\title{
The association of antidiabetic medications and Mini-Mental State Examination scores in patients with diabetes and dementia
}

\author{
Juraj Secnik ${ }^{1,2^{*}}$, Hong Xu ${ }^{1,3}$, Emilia Schwertner ${ }^{1}$, Niklas Hammar ${ }^{4}$, Michael Alvarsson ${ }^{5}$, Bengt Winblad ${ }^{6,7}$,
} Maria Eriksdotter ${ }^{1,7}$, Sara Garcia-Ptacek ${ }^{1,7}$ and Dorota Religa ${ }^{1,7}$

\begin{abstract}
Background: The effect of antidiabetic medication on cognitive function is unclear. We analyzed the association between five antidiabetic drugs and change in Mini-Mental State Examination (MMSE) scores in patients with diabetes and dementia.

Methods: Using the Swedish Dementia Registry and four supplementary Swedish registers/databases, we identified 1873 patients (4732 observations) with diagnosis of type 2 diabetes (diabetes) and Alzheimer's disease or mixedpathology dementia who were followed up at least once after dementia diagnosis. Use of metformin, insulin, sulfonylurea, thiazolidinediones (TZD), and dipeptidyl-peptidase-4 inhibitors (DPP-4i) was identified at baseline. Prevalentuser, incident-user, and drug-drug cohorts were sampled, and propensity-score matching was used to analyze comparable subjects. Beta coefficients with 95\% confidence intervals (Cl) from the random intercept and slope linear mixed-effects models determined the association between the use of antidiabetic medications and decline in MMSE score points between the follow-ups. Inverse-probability weighting was used to account for patient dropout.

Results: Compared to non-users, prevalent users of metformin (beta 0.89, 95\% Cl 0.44; 1.33) and DPP-4i (0.72, 0.06; 1.37) experienced a slower cognitive decline with time. Secondly, compared to DPP-4i, the use of insulin ( -1.00 , $-1.95 ;-0.04)$ and sulfonylureas $(-1.19 ;-2.33$; - 0.04) was associated with larger point-wise decrements in MMSE with annual intervals.
\end{abstract}

Conclusions: In this large cohort of patients with diabetes and dementia, the use of metformin and DPP-4i was associated with a slower decline in MMSE scores. Further examination of the cognitive effects of metformin and incretinbased medications is warranted.

Keywords: Diabetes, Dementia, Antidiabetics, Metformin, DPP-4i, MMSE

\section{Background}

Diabetes is a complex risk factor for cognitive deterioration and a common comorbidity in dementia [1, 2]. In addition, multiple antidiabetic drugs have been evaluated

\footnotetext{
*Correspondence: juraj.secnik@ki.se

1 Division of Clinical Geriatrics, Center for Alzheimer Research,

Department of Neurobiology, Care Sciences and Society, Karolinska Institutet, Neo, Blickagången 16, 14152 Huddinge, Sweden

Full list of author information is available at the end of the article
}

in randomized and observational settings for putative cognitive properties. Specifically, metformin has been associated with protection against overall dementia risk and memory decline $[3,4]$; however, some studies suggest an even opposite relationship [5], possibly due to vitamin B12 deficiency in long-term users [6] contributing to neurodegeneration.

Moreover, the role of cerebral insulin gained recognition in the last decade [7]; however, neither intranasal

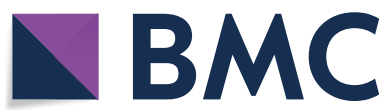

(c) The Author(s) 2021. Open Access This article is licensed under a Creative Commons Attribution 4.0 International License, which permits use, sharing, adaptation, distribution and reproduction in any medium or format, as long as you give appropriate credit to the original author(s) and the source, provide a link to the Creative Commons licence, and indicate if changes were made. The images or other third party material in this article are included in the article's Creative Commons licence, unless indicated otherwise in a credit line to the material. If material is not included in the article's Creative Commons licence and your intended use is not permitted by statutory regulation or exceeds the permitted use, you will need to obtain permission directly from the copyright holder. To view a copy of this licence, visit http://creativecommons.org/licenses/by/4.0/. The Creative Commons Public Domain Dedication waiver (http://creativeco mmons.org/publicdomain/zero/1.0/) applies to the data made available in this article, unless otherwise stated in a credit line to the data. 
nor systemic insulin administration has significantly improved cognitive functioning $[8,9]$. Similarly, the studies on sulfonylurea derivates have found either no association [10] or higher relative risk of dementia compared to metformin [11]. On the other hand, both sulfonylurea and systemic insulin treatment are associated with the risk of hypoglycemia [12], which may contribute to dementia incidence [13]. Moreover, manifest dementia is an independent predictor of severe hypoglycemia [14], as cognitive health is a prerequisite for proper diabetes self-management [15].

Thiazolidinediones (TZD) have not exhibited cognitive benefit in a randomized controlled setting [16]; moreover, faster memory decline was observed in TZD users among patients with Alzheimer's disease (AD) [4].

Among the drugs affecting the incretin system, the glucagon-like peptide-1 analogues (GLP-1a) increased cerebral glucose metabolism in a small randomized controlled trial [17], while multiple neuroprotective mechanisms have been suggested in animal models of AD [18]. Second, the use of dipeptidyl-peptidase-4 inhibitors (DPP-4i) was cognitively protective in observational [4, 19] but not in interventional studies [20].

The potential recommendations for cognitive performance are further complicated by the applications of specific antidiabetic drugs in the course of diabetes (diabetes control, complications, application method, patient preference, etc.).

In summary, despite robust relationships between diabetes and dementia, it is unclear whether some antidiabetic medications provide cognitive benefit compared to others. Moreover, studies evaluating the rate of cognitive decline in patients with established dementia are scarce. Due to the absence of disease-modifying dementia treatment, good management of comorbidities can play a major role in preserving residual cognitive functioning in dementia patients.

The aim of this study was to compare the change in Mini-Mental State Examination (MMSE) scores among users of five antidiabetic drug groups in a large cohort of patients with diabetes and AD or mixed-pathology dementia (MixDem).

\section{Material and methods}

This was a prospective open-cohort study with data originating from five Swedish registries/databases. Swedish personal identification number (personnummer) was used to merge information across data sources, with the National Board of Health and Welfare and Statistics Sweden performing the merge and data anonymization. Registers and data are described below.

\section{Swedish Dementia Registry (SveDem)}

SveDem was established in 2007 with the aim to register all patients with dementia in Sweden at the time of diagnosis [21, 22]. SveDem comprises information on clinical determinants (e.g., MMSE), demography (e.g., age and living arrangements), community support (e.g., daycare), and common pharmacological treatment [21]. Dementia diagnoses are coded using ICD-10 and comprise AD, MixDem (both $\mathrm{AD}$ and vascular pathology present), vascular dementia, Lewy body dementias, frontotemporal dementia, unspecified, and other dementia types. Patients are followed up on an annual basis with clinical examination including cognitive assessment using MMSE.

\section{Dementia}

Out of the 80,004 patients registered in SveDem between May 1, 2007, and October 16, 2018, we included only patients with a diagnosis of AD or MixDem who were also diagnosed with diabetes (diabetes sections below) and had been followed up at least once. SveDem provided data on demography (age, sex), type of dementia, cohabitation, and baseline and follow-up dates with MMSE scores (the outcome). After applying sample restrictions, the final cohort consisted of 1873 patients with 4732 observations (baseline and follow-ups; supplementary figure 1). This population was then used to sample propensity-score exposure-matched pairs for the analyses.

\section{Swedish National Patient Register (Patient Register)}

The Patient Register provided records on inpatient diagnoses since 1998 and specialized outpatient visits since 2001 [23] until December 31, 2017. Diagnoses were coded according to the 10th version of the International Classification of Diseases (ICD-10) [24].

\section{Diabetes mellitus}

Diagnosis of diabetes was determined when the ICD-10 codes E10-E14 occurred in the Patient Register or antidiabetic drug dispensation (Anatomical Therapeutic and Chemical [ATC] classification code A10) was observed in the Swedish Prescribed Drug Register (Drug Register) prior to and including the date of dementia diagnosis. Subsequently, diabetes was grouped into three typestype 1 diabetes, type 2 diabetes, and other/unspecified diabetes (for details on extraction and coding, see supplementary algorithm 1). Only patients with type 2 diabetes and other/unspecified types were included for the analyses.

Baseline diabetes duration was based on the difference between the date of dementia diagnosis and the date of the earliest record of diabetes-either in the Patient 
Register where the diagnosis of diabetes occurred or the earliest dispensation date of ATC code A10 from the Drug Register, whichever came first.

\section{Comorbidities}

To adjust for the effect of additional chronic diseases, we created a baseline comorbidity index as described by Charlson et al. [25], using the algorithm described by Quan et al. [26] as a weighted sum of diagnosed chronic disorders up to and including the date of dementia diagnosis. Renal disease was not included in the index and was extracted as a separate variable. Diabetes and dementia variables were omitted from the index.

\section{Longitudinal integrated database for health insurance and labour market studies (LISA)}

LISA is an administrative database covering the adult Swedish population since 1990 and provides high-quality information on major socioeconomic characteristics (sick leave, disability, pensions, etc.) [27].

\section{Disposable income}

To take baseline socioeconomic position into account, disposable income at the time of dementia diagnosis inflated on the 2019 value of Consumer Price Index was extracted from LISA and categorized into low-, middle-, and high-income groups with 33rd and 66th percentiles used as cut-offs.

\section{Swedish Prescribed Drug Register}

The Drug Register was established in 2005 and stores information on all dispensed drug prescriptions at Swedish pharmacies using ATC coding [28]. Medication dispensation data (=prescription fills) were extracted from the start of the register until December 31, 2018.

\section{Diabetes mellitus}

ATC codes A10 (drugs used in diabetes), A10A (insulins), and $\mathrm{A} 10 \mathrm{~B}$ (blood glucose-lowering drugs excluding insulin) before and after dementia diagnosis extracted from the Drug Register were used in combination with the Patient Register to identify overall diabetes prevalence and duration and to classify diabetes types (see supplementary algorithm 1 ).

\section{Antidiabetic medications}

Data on antidiabetic drug classes was extracted from the Drug Register according to the following ATC codes-insulin (A10A), metformin (A10BA02), sulfonylurea derivates (SU; $\mathrm{A} 10 \mathrm{BB})$, thiazolidinediones (A10BG), dipeptidyl-peptidase-4 inhibitors (A10BH), glucagon-like peptide-1 analogues (A10BJ), and sodiumglucose co-transporter-2 inhibitors (SGLT-2i; A10BK).
Dispensations of the individual medication classes were extracted on a yearly basis prior to the date of dementia diagnosis date. GLP-1a and SGLT-2i could not be directly analyzed due to too few users; however, their use was matched on in the propensity-score matching. Two frameworks for exposure were created.

First, patients were grouped into baseline users and non-users of individual medications according to the incident and prevalent exposure design. A subject was an incident user if a dispensation of medication was observed in the 1-year period prior to and including the date of dementia diagnosis, without a record of medication dispensation prior to the 1-year period. Complementary incident non-user was a subject without a dispensation in neither the 1 -year period nor at any time before the diagnosis of dementia. Prevalent use was based on the presence/absence of at least one medication dispensation prior to the diagnosis of dementia.

Second, we compared non-metformin antidiabetic medications directly. For example, to compare baseline insulin vs baseline sulfonylurea, patients exposed to insulin treatment at least once prior to diagnosis of dementia while never having sulfonylurea prior to diagnosis of dementia constituted one group, while subjects who had sulfonylurea and never had insulin constituted the comparison group. Due to the low number of possible pairs, we could only analyze prevalent users (see the section "Statistical analysis"). Metformin was not compared directly to other medications due to its specific position as first-line therapy and very frequent use in the cohort, thus lacking a sufficient number of patients in the nevermetformin control group.

Subsequently, propensity-score matching on baseline exposure assignment was performed to produce comparable user/non-user and user/other-user pairs. Afterwards, the associations between antidiabetic drug usage and the change in post-dementia MMSE scores were assessed in intention-to-treat analyses.

\section{Supplementary medication}

Dispensations of antihypertensive (ATC codes C02, C07, C08, and C09), hypolipidemic (statins, C10AA), antithrombotic (B01), antipsychotic (N05A), antidepressant drugs (N06A), and cholinesterase inhibitors (N06DA) were extracted up to 3 years prior to and including the date of dementia diagnosis as recorded by the Drug Register.

\section{Swedish Cause of Death Register (Death Register)}

The records in the Death Register begin from the year 1952 and are the basis for official statistics on causes of death in Sweden [29]. The register's purpose is to 
describe the development of national all-cause and specific-cause mortality.

\begin{abstract}
Mortality
We extracted the information from the Death Register since its initiation until October 16, 2018-the end of the study follow-up. Overall mortality was considered if a valid record (patient death dated after the date of dementia diagnosis) was present. The information on mortality was used to determine censoring and to account for patient dropout.
\end{abstract}

\section{Study sample selection}

The original cohort consisted of 80,004 patients diagnosed with dementia and registered to SveDem until October 16, 2018. SveDem data were linked with other data sources and several selection criteria were applied, mainly excluding incorrect or missing baseline information. Afterwards, 12,396 patients who had diagnosis of diabetes and dementia at baseline were identified. After including only patients with at least one follow-up, exclusions of other dementia types than AD or MixDem, patients with diabetes type 1 and baseline severe dementia (MMSE < 10; to avoid floor effects), the final cohort consisted of 1873 patients and 4732 observations (supplementary figure 1).

\section{Statistical analysis Propensity-score matching}

From the whole cohort of 1873 subjects with diabetes and dementia, we sampled PS-matched comparable pairs of users/non-users and users/other-users of antidiabetic medications as per the framework described above. The 1:1 and 1:4 nearest-neighbor matching with 0.1 caliper of the logit of the propensity score was used. Baseline characteristics used to generate PS included age at dementia diagnosis; sex; cohabitation; dementia type; Charlson comorbidity score; renal disease; diabetes type and duration; income category; use of statins; antihypertensive, antithrombotic, antipsychotic, and antidepressant drugs; cholinesterase inhibitors; use of other glucose-lowering drugs apart from insulin (as a summary measure user/ non-user); and use of insulin (omitted in insulin analyses) prior to dementia diagnosis. Matching was less extensive in situations with few available exposed subjects (to uphold the 10 subjects per predictor rule), and matching priority was given to prognostic characteristics. Matching ratio 1:4 was used in the prevalent-user analyses of DPP-4i and TZD, all incident-user analyses, and comparisons of insulin vs DPP-4i, insulin vs TZD, sulfonylurea vs TZD, and sulfonylurea vs DPP-4i. Other cohorts were matched using a 1:1 ratio.
For the incident-user analyses, we identified the following user/non-user cohorts: 101 metformin users vs 277 non-users, 66 insulin users vs 263 non-users, and 37 sulfonylurea users vs 147 non-users. Prevalent-user analyses were based on 514 metformin user/non-user pairs, 543 insulin pairs, 640 sulfonylurea pairs, 67 TZD users vs 260 non-users, and 103 DPP-4i users vs 389 non-users (Table 1).

In the non-metformin antidiabetic drug comparisons, 259 pairs of insulin vs sulfonylurea users were generated, 138 insulin vs 35 TZD users, 199 insulin vs 51 DPP-4i users, 111 sulfonylurea vs 31 TZD users, 141 sulfonylurea vs 38 DPP- 4 i users, and 45 DPP- $4 \mathrm{i}$ vs TZD pairs. The baseline differences between matched cohorts as well as the number eligible for individual matchings from the original cohort are summarized in Table 1 and supplementary tables 1 and 3.

\section{Patient dropout-inverse-probability weighting}

Patient dropout was determined, if the patient had neither died nor did the study end occur in the year following the last observed MMSE. The last observed MMSE was defined as the last observed MMSE measurement, provided the time difference between the last observed MMSE examination and previous MMSE examination was more than 9 months to reflect the yearly schedule of follow-ups. If the difference was less than 9 months, the 1-year period for dropout was assessed from the previous date of MMSE measurement. Using this definition, the overall dropout rate was high (1382 patients [73.8\%]; supplementary table 2); thus, we weighted the analyses using inverse probability of remaining in the study based on the MMSE scores in the previous observation and time since baseline. We used the same process as described by Handels and colleagues [30]: first computing the probability of dropout in the next observation, then defining cumulative probabilities of remaining in the study, and finally producing an inverse of the cumulative probabilitiesinverse-probability weights of remaining in the study. The weights were unstabilized and truncated at 100 if the weight was above the 99th percentile of the weight distribution to avoid the models being dominated by few large weights.

\section{Descriptive statistics}

Differences in baseline characteristics between the matched pairs of users and non-users of antidiabetic medications were assessed using chi-square, independent samples $t$-test, and their non-parametric equivalents. Standardized mean differences $(S M D s)$ were used to assess balance in the propensity-score-matched cohorts. 


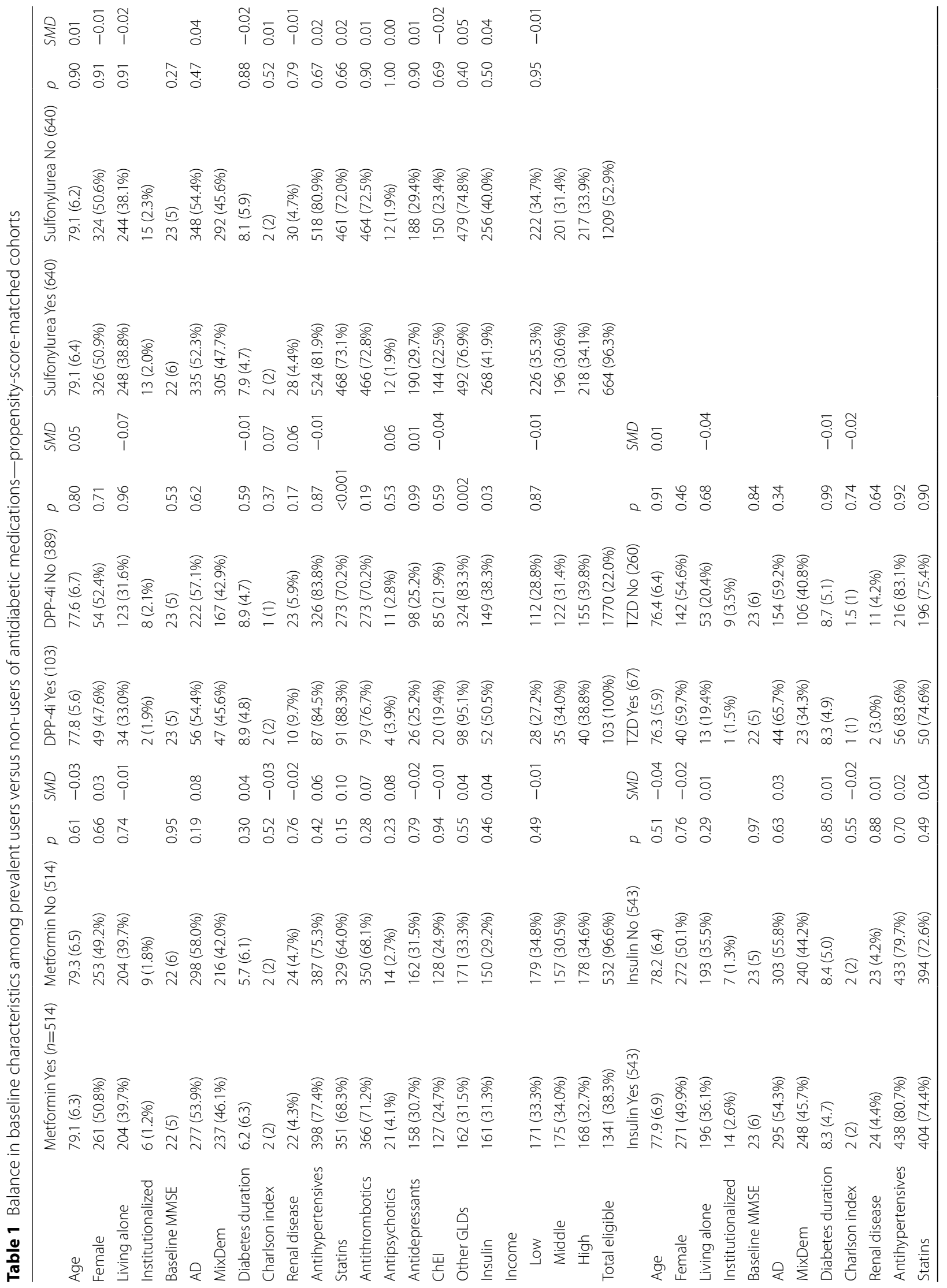




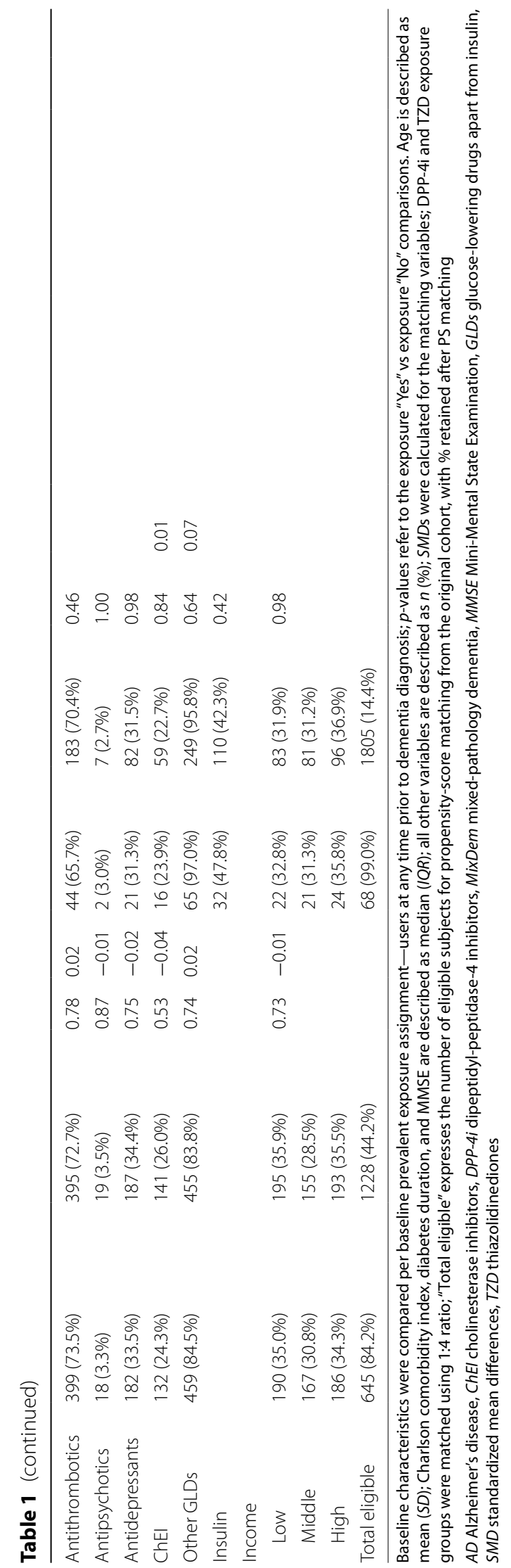




\section{MMSE change-mixed-effects models}

In the PS-matched cohorts, random-slope linear mixedeffects models were used to determine the beta coefficients with $95 \%$ confidence intervals of the associations between the use of antidiabetic medications and change in MMSE scores with time in years. Fixed effects in the model included only the interaction between the users/ non-users or users/other users of antidiabetic medication with time. Random effects were included for subjects and time (intercept and slope). Three mixed-effects models were designed in the propensity-score-matched cohorts-unweighted analysis, inverse-probabilityweighted analysis, and weighted analysis in the imputed dataset. Residual baseline differences between the matched cohorts were adjusted for and kept in the models if both the association with MMSE and 10\% change in the main exposure were significant. Subjects with missing information in matching or weighting variables were excluded prior to analysis (see supplementary figure 1).

Data were analyzed using Stata v16 (Stata Statistical Software: Release 16. StataCorp LLC, College Station, TX) and R version 4.0.0 [31] with package "MatchIt."

\section{Sensitivity analysis_-imputed dataset}

To model the ideal scenario with yearly follow-ups, we have imputed MMSE scores based on the slope between the observed MMSE measurements and time from baseline. Missing scores were imputed using multivariate multiple imputation with chained equations. Imputations were done only in observations with missing yearly follow-ups between observed MMSE (e.g., observed baseline and year 3) and not performed beyond observed MMSE. Afterwards, the inverse-probability-weighted mixed-effects models were performed in the imputed dataset.

\section{Results}

\section{Baseline differences}

The differences between the matched cohorts are summarized in Table 1 (prevalent use) and supplementary table 1 (incident use) and 3 (drug vs drug comparisons).

The PS matching provided balanced exposure groups, except for few differences. DPP-4i users were more frequently exposed to statins in all cohorts $(88.3 \%$ vs $70.2 \%$ in prevalent users; $90.2 \%$ vs $75.9 \%$ in DPP-4i vs insulin; $97.4 \%$ vs $75.2 \%$ DPP- $4 \mathrm{i}$ vs sulfonylurea). Incident insulin users were less commonly treated with antidepressants (15.2\% vs $34.6 \%)$. Finally, incident metformin users had significantly shorter diabetes duration ( 0.8 vs 3.5 years). All residual differences were adjusted for in the mixed models.

\section{MMSE and antidiabetic medications}

The results from the random-slope linear mixedeffects models are summarized in Tables 2 and 3 . In the weighted user vs non-user analyses, the use of metformin was associated with a slower annual decline in MMSE compared to non-users, specifically 0.89 points slower in prevalent users vs non-users $(\beta 0.89,95 \% C I 0.44 ; 1.33)$, however not in incident-user weighted analysis $(0.70$, -0.16 ; 1.56). Similar association was observed in the unweighted $(0.67,0.35 ; 0.99)$ and imputed analyses $(0.88$, $0.44 ; 1.32$ ). The prevalent use of DPP-4i was associated with a slower annual MMSE decline compared to nonusers in the weighted $(0.72,0.06 ; 1.37)$ and imputed $(0.70$, $0.03 ; 1.37)$ analyses, but not statistically significantly in the unweighted analysis $(0.53,-0.02 ; 1.09 ; p=0.06)$.

Figure 1 visualizes the main observed trends over time in the annual MMSE decline in users of metformin, DPP$4 \mathrm{i}$, and comparisons between insulin and sulfonylurea and insulin and DPP-4i based on the weighted analyses.

In the head-to-head comparisons of the non-metformin antidiabetic medications, the use of sulfonylureas was associated with an accelerated MMSE decline compared to DPP-4i users $(-1.10,-2.12 ;-0.08$; weighted analyses), with similar findings in the unweighted (-1.07; $-2.10 ;-0.05)$ and imputed models $(-1.07,-2.12$; $-0.02)$. Similarly, insulin users experienced an accelerated cognitive decline compared to DPP-4i users $(-1.00$, $-1.95 ;-0.04)$ when weighted for dropout.

\section{Discussion}

In this large cohort of patients with diabetes and dementia, the use of metformin and DPP-4i was associated with a slower decline in MMSE scores in time compared to non-users. Moreover, patients with dementia using DPP$4 \mathrm{i}$ experienced a slower decline in MMSE scores compared to sulfonylurea and insulin users.

The overall (prevalent) use of metformin was associated with a slower decline in MMSE compared to nonusers, suggesting either a well-managed diabetes in metformin users or a direct neuroprotective effect of metformin. While lower dementia risk was observed in metformin users in multiple studies [11, 32, 33], MMSE was unaffected in a 2018 meta-analysis [3]. On the other hand, metformin was recently associated with protection against memory decline, however only in patients with normal cognition and without ApoE- $\varepsilon 4$ burden [4]. While we could not stratify on ApoE genotype, we analyzed metformin initiation, which failed to provide significant benefit to users, despite the shorter diabetes duration. Metformin's role in the AD pathological process is uncertain, with both neuroprotective [34-36] and pathologyaccelerating properties $[37,38]$ reported in non-human studies. Recently, Wu and colleagues suggested that 
Table 2 Annual point change in post-dementia Mini-Mental State Examination scores associated with the use of antidiabetic medications

\begin{tabular}{|c|c|c|c|c|c|c|c|c|c|}
\hline & \multicolumn{3}{|c|}{$\begin{array}{l}\text { Unweighted analysis User vs non- } \\
\text { user }\end{array}$} & \multicolumn{3}{|c|}{ Weighted analysis User vs non-user } & \multicolumn{3}{|c|}{$\begin{array}{l}\text { Imputed weighted analysis User vs } \\
\text { non-user }\end{array}$} \\
\hline & $\beta$ coefficients $(95 \% C l)$ & $Z$ & $p$ & $\beta$ coefficients $(95 \% \mathrm{Cl})$ & $Z$ & $p$ & $\beta$ coefficients $(95 \% C l)$ & $z$ & $p$ \\
\hline \multicolumn{10}{|c|}{ Propensity-score-matched cohorts } \\
\hline $\begin{array}{l}\text { Metformin } \times \text { time } \\
\text { (incident users) }\end{array}$ & $0.38(-0.20 ; 0.95)$ & 1.28 & 0.20 & $0.70(-0.16 ; 1.56)$ & 1.59 & 0.11 & $0.53(-0.31 ; 1.37)$ & 1.23 & 0.22 \\
\hline $\begin{array}{l}\text { Metformin } \times \text { time } \\
\text { (prevalent users) }\end{array}$ & $0.67(0.35 ; 0.99)$ & 4.15 & $<0.001$ & $0.89(0.44 ; 1.33)$ & 3.93 & $<0.001$ & $0.88(0.44 ; 1.32)$ & 3.91 & $<0.001$ \\
\hline $\begin{array}{l}\text { Insulin } x \text { time (inci- } \\
\text { dent users) }\end{array}$ & $-0.25(-0.83 ; 0.33)$ & -0.84 & 0.40 & $-0.28(-1.22 ; 0.67)$ & -0.57 & 0.57 & $-0.37(-1.34 ; 0.60)$ & -0.74 & 0.46 \\
\hline $\begin{array}{l}\text { Insulin } x \text { time (preva- } \\
\text { lent users) }\end{array}$ & $-0.06(-0.37 ; 0.25)$ & -0.41 & 0.68 & $-0.03(-0.48 ; 0.42)$ & -0.13 & 0.90 & $-0.12(-0.56 ; 0.32)$ & -0.54 & 0.59 \\
\hline $\begin{array}{l}\text { Sulfonylurea } x \text { time } \\
\text { (incident users) }\end{array}$ & $0.05(-0.84 ; 0.93)$ & 0.10 & 0.92 & $-0.10(-1.38 ; 1.17)$ & -0.16 & 0.87 & $-0.19(-1.48 ; 1.11)$ & -0.28 & 0.78 \\
\hline $\begin{array}{l}\text { Sulfonylurea } x \text { time } \\
\text { (prevalent users) }\end{array}$ & $-0.08(-0.36 ; 0.20)$ & -0.55 & 0.59 & $-0.11(-0.53 ; 0.31)$ & -0.52 & 0.60 & $-0.13(-0.56 ; 0.29)$ & -0.62 & 0.53 \\
\hline $\begin{array}{l}\text { TZD x time (prevalent } \\
\text { users) }\end{array}$ & $0.12(-0.49 ; 0.73)$ & 0.38 & 0.71 & $0.14(-0.76 ; 1.04)$ & 0.30 & 0.76 & $0.04(-0.91 ; 0.98)$ & 0.07 & 0.94 \\
\hline $\begin{array}{l}\text { DPP-4i } \times \text { time (preva- } \\
\text { lent users) }\end{array}$ & $0.53(-0.02 ; 1.09)$ & 1.64 & 0.06 & $0.72(0.06 ; 1.37)$ & 2.14 & 0.032 & $0.70(0.03 ; 1.37)$ & 2.04 & 0.041 \\
\hline
\end{tabular}

Beta coefficients were acquired from random-slopes linear mixed-effects models. Weighting was performed for the inverse probability of remaining in the study. Sulfonylurea and DPP-4i incident-user analyses and GLP-1a and SGLT-2i prevalent-user analyses were performed in 1:4 PS-matched cohorts. Time scale is expressed in years

DPP-4i dipeptidyl-peptidase-4 inhibitors, GLP-1a glucagon-like peptide-1 analogues, SGLT-2i sodium-glucose co-transporter-2 inhibitors

Table 3 Comparisons between non-metformin antidiabetic drugs and annual point change in Mini-Mental State Examination scores

\begin{tabular}{|c|c|c|c|c|c|c|c|c|c|}
\hline & \multicolumn{3}{|c|}{ Unweighted analysis Drug vs drug } & \multicolumn{3}{|c|}{ Weighted analysis Drug vs drug } & \multicolumn{3}{|c|}{$\begin{array}{l}\text { Imputed weighted analysis Drug } \\
\text { vs drug }\end{array}$} \\
\hline & $\beta$ coefficients $(95 \% \mathrm{Cl})$ & $Z$ & $p$ & $\beta$ coefficients $(95 \% \mathrm{Cl})$ & $Z$ & $p$ & $\beta$ coefficients $(95 \% \mathrm{Cl})$ & $z$ & $p$ \\
\hline \multicolumn{10}{|c|}{ Propensity-score-matched cohorts } \\
\hline $\begin{array}{l}\text { Insulin vs sulfonylurea } x \\
\text { time (259 user pairs) }\end{array}$ & $0.14(-0.28 ; 0.56)$ & 0.65 & 0.52 & $0.29(-0.35 ; 0.92)$ & 0.89 & 0.37 & $0.29(-0.32 ; 0.90)$ & 0.93 & 0.35 \\
\hline $\begin{array}{l}\text { Insulin vs TZD x time (138 } \\
\text { users vs } 35 \text { users) }\end{array}$ & $-0.23(-1.04 ; 0.58)$ & -0.56 & 0.58 & $-0.86(-1.95 ; 0.24)$ & -1.53 & 0.13 & $-0.90(-2.03 ; 0.23)$ & -1.56 & 0.12 \\
\hline $\begin{array}{l}\text { Insulin vs DPP-4i x time } \\
\text { (199 users vs } 51 \text { users) }\end{array}$ & $-0.82(-1.72 ; 0.07)$ & -1.80 & 0.07 & $-1.00(-1.95 ;-0.04)$ & -2.04 & 0.042 & $-0.93(-1.87 ; 0.01)$ & -1.94 & 0.053 \\
\hline $\begin{array}{l}\text { Sulfonylurea vs DPP- } 4 \mathrm{ix} \\
\text { time ( } 141 \text { users vs } 38 \text { users) }\end{array}$ & $-1.07(-2.10 ;-0.05)$ & -2.05 & 0.040 & $-1.19(-2.33 ;-0.04)$ & -2.05 & 0.041 & $-1.15(-2.33 ; 0.02)$ & -1.93 & 0.054 \\
\hline $\begin{array}{l}\text { Sulfonylurea vs TZD x } \\
\text { time (111 users vs } 31 \text { users) }\end{array}$ & $-0.62(-1.58 ; 0.34)$ & -1.26 & 0.21 & $-1.00(-2.34 ; 0.35)$ & -1.46 & 0.15 & $-0.98(-2.34 ; 0.38)$ & -1.41 & 0.16 \\
\hline $\begin{array}{l}\text { DPP-4i vs TZD x time ( } 45 \\
\text { user pairs) }\end{array}$ & $0.15(-0.78 ; 1.08)$ & 0.31 & 0.76 & $-0.19(-1.48 ; 1.10)$ & -0.29 & 0.77 & $-0.16(-1.48 ; 1.15)$ & -0.25 & 0.81 \\
\hline
\end{tabular}

Based on random-slopes linear mixed-effects models. Medication use was assigned, if a patient had a dispensation of the primary medication prior to diagnosis of dementia, while never having a dispensation of the compared medication prior to diagnosis of dementia and vice versa. Weighting was performed for the inverse probability of remaining in the study. Time was expressed in years

metformin's putative neuroprotection may be limited to preclinical AD stages, whereas its later use may contribute to AD pathology [4]. In our cohort, we conclude metformin's cognitive effect as neutral to positive; however, we lacked a comparable number of never-exposed to metformin for prevalent users, and this analysis drove the protective association. Moreover, the comparison group of metformin non-users comprised a non-negligible number of subjects without any medication for diabetes, which likely affected the association even after thorough matching; however, it is not clear in which direction. Possibly, metformin's molecular changes may not be directly 

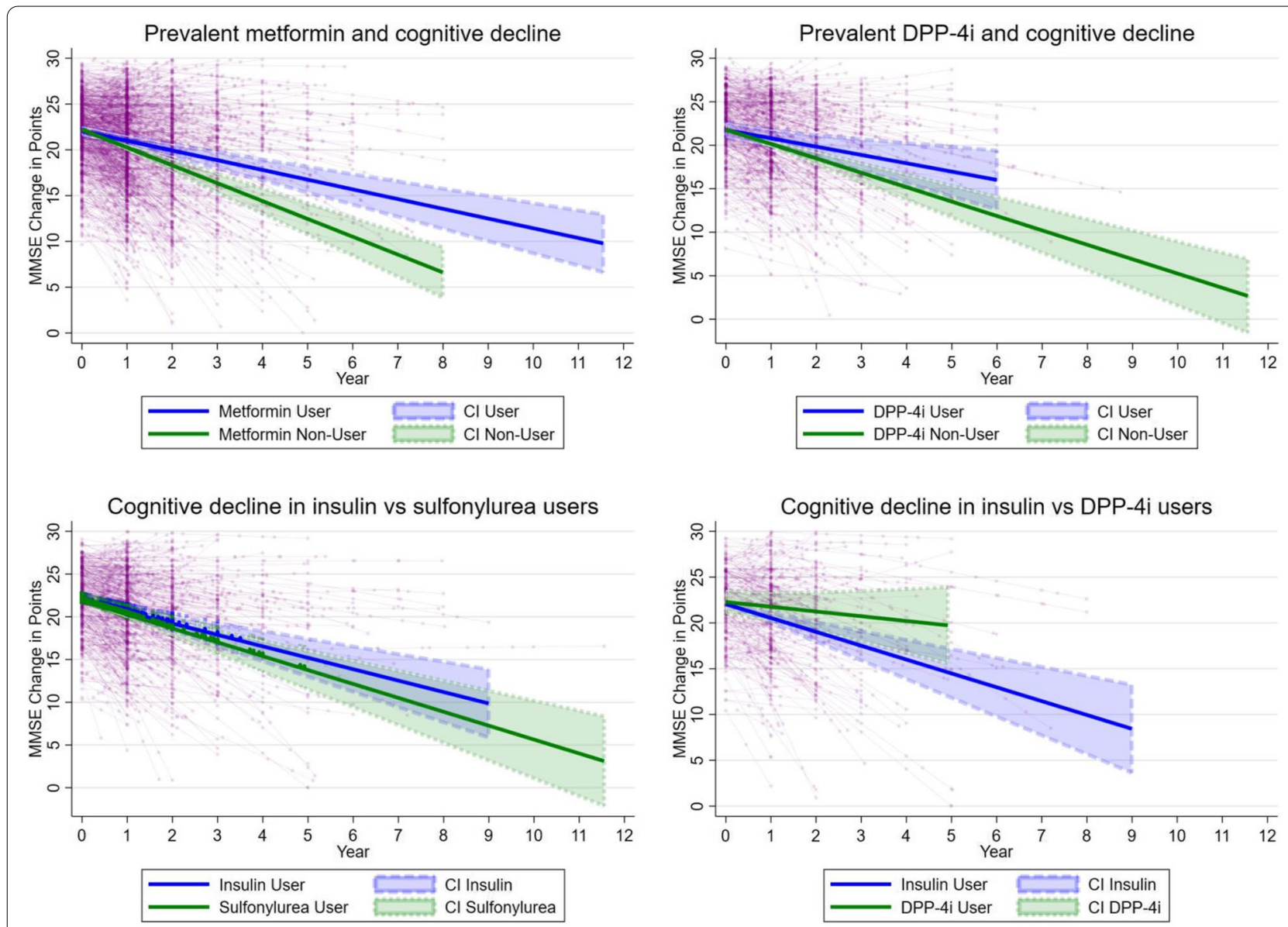

Fig. 1 Linear changes in MMSE scores associated with the use of metformin, DPP-4i, insulin, and sulfonylurea exposure among patients with dementia and diabetes. Figures are based on weighted prevalent-user analyses; DPP-4i, dipeptidyl-peptidase-4 inhibitors; $\mathrm{Cl}$, confidence interval; MMSE, Mini-Mental State Examination; year represents time after baseline; thin lines represent MMSE change in individual subjects (circles) in time, and thick lines represent estimated MMSE change derived from the mixed models

reflected on a robust point-based MMSE score; however, if metformin would exhibit serious cognitive harm, this would likely be noticed between the follow-ups. Overall, a life-course approach combined with the evaluation of individual cognitive domains would be valuable to deconstruct metformin's neuronal properties.

Importantly, the prevalent users of DPP-4i experienced a significantly slower decline in MMSE scores compared to non-users. Among incretin-based therapies, DPP$4 \mathrm{i}$ are frequently used in Swedish patients with dementia [39], possibly due to the oral application and neutral weight effects. In observational studies, DPP-4i were associated with a lower risk of dementia [10,40] and improvements in immediate and delayed memory in patients with established AD [4]. On the other hand, a recent randomized trial of linagliptin failed to show cognitive benefit in high-risk albeit dementia-free type 2 diabetes patients [20].
In our cohort, DPP-4i exhibited a 0.7-point slower MMSE decline in users vs non-users, corroborating the findings by Isik and colleagues [19]. Similarly, Rizzo and colleagues concluded higher MMSE among DPP-4i-metformin therapy compared to sulfonylurea-metformin after 2 years [41]; however, patients with dementia were excluded making the comparison difficult. Moreover, the addition of DPP-4i vildagliptin to metformin was associated with higher MMSE at 6 months compared to metformin alone, but the study was underpowered for multivariate analyses [42].

DPP-4i users specifically experienced a slower MMSE decline compared to sulfonylurea and insulin users. Conceptually, patients using sulfonylureas and insulin may have been at greater risk of hypoglycemia (DPP-4i have negligible hypoglycemia risk) [12]; unfortunately, we could not test such hypothesis. On the other hand, Cukierman-Yaffe and colleagues have recently contested the connection between severe hypoglycemia and cognitive 
decline; however, it is unclear whether this extends to dementia patients [43].

DPP-4i exhibit their antidiabetic effect in a glucosedependent manner by increasing the endogenous GLP-1 through inhibition of its breakdown [12]; however, their potency is lower compared to GLP-1a [44]. Interestingly, the preclinical studies on GLP-1's role in cerebral metabolism concluded amelioration of oxidative stress, growth factor, and insulin signaling in AD pathology [18]. DPP4i could possibly provide direct neuronal benefit; however, enhancing cerebral signaling through increasing the pool of GLP-1 is more likely [45] as the DPP-4i transfer through the blood-brain barrier is limited in comparison to GLP-1a [46].

Unfortunately, our study lacked the power to analyze GLP-1a users, which would strengthen the argument for the incretin-based therapies if a similar association was observed.

In general, the available evidence suggests that the protection associated with incretin therapies may be limited to later phases of neurodegeneration.

Despite the encouraging results for metformin and DPP-4i, the inter-follow-up MMSE change was rather modest (approximately 1 point) and the minimal clinically important decline in MMSE was recently estimated at around $2-3$ points [47]. If accurate, the difference in DPP-4i and metformin users vs non-users should be observable approximately at the third-year follow-up. On the other hand, any preservation of cognitive functioning associated with the use of established antidiabetic drugs should be considered valuable, especially in the absence of disease-modifying therapy.

Neither sulfonylurea nor insulin was associated with a significant change in MMSE scores compared to nonusers, while a similar decline was observed when directly comparing the two drugs or compared to TZD.

Sulfonylurea's neurophysiological properties are not entirely clear, as some evidence suggests a higher risk of dementia in comparison to metformin [11, 32], or no association $[10,48]$. In cognitive functioning, sulfonylureas have not modified either delayed or immediate memory [4], while a larger, albeit inconsistent global cognitive change was observed in sulfonylurea users in a pooled meta-analysis [48]. We provide robust evidence that sulfonylurea use does not affect global cognition in patients with $\mathrm{AD}$ or MixDem.

On the other hand, insulin treatment has a specific position in the treatment of type 2 diabetes in Sweden, where its use is prioritized in the guidelines [49] and common even in patients with dementia [39]. While insulin use was associated with a higher risk of all-cause dementia and a modestly larger decline in global cognition [48], the association was not found in other studies
$[9,10]$. In line with our findings, Cukierman-Yaffe and colleagues reported no effect of basal insulin on MMSE scores; however, the population was younger and cognitively unimpaired at baseline [9]. Thus, insulin indeed may be cognitively neutral; however, further research is needed to clarify if hypoglycemia may balance out some cognitive protection, especially in a region with frequent insulin usage.

We have not observed any substantial effect on global cognition in patients using TZD, corroborating the randomized controlled trials on rosiglitazone [16] and pioglitazone [50]. While TZD may accelerate memory decline in ApoE- $\varepsilon 4$ non-carriers with $\mathrm{AD}$ and diabetes [4], we did not have access to such data. Currently, the only approved TZD in Europe is pioglitazone; however, a careful patient selection is necessary prior to drug initiation due to a higher risk of edema, heart failure, and fractures [12]. Overall, the use of TZD in elderly patients with dementia exhibited neither cognitive protection nor harm in our cohort.

In conclusion, metformin and DPP-4i had a positive effect on global cognition measured by MMSE in patients with diabetes and dementia. Further research should focus on the putative neurocognitive properties of incretin and biguanide therapy.

\section{Limitations}

One of the main limitations is the observational nature of the study, precluding us from concluding causal relationships. Second, SveDem is a real-world database and thus suffers from significant patient dropout, which cannot be controlled for in the data collection phase. Conversely, we have addressed this issue of selective dropouts by weighting on the inverse probability of remaining in the study, which produced reasonable estimates and was previously used in the SveDem setting [30]. MMSE has known ceiling and floor effects; however, the median baseline MMSE score was 21 points (18 and 24 being the 25th and 75th percentile) and patients with baseline MMSE below 10 points were excluded. Most patients had been living with diabetes for several years; thus, there were insufficient incident-users for more recent medications and for head-to-head drug comparisons. While we had a plethora of data on confounding, information on two principal confounders was lacking-glycemic markers (such as HbA1c) and the ApoE genotype. The absence of HbA1c likely skewed some of the results in favor of metforminbeing the first-line therapy and representing a group of patients with an overall shorter course of diabetes and fewer complications. On the other hand, the insufficient data on hypoglycemic episodes may have confounded the results of insulin and sulfonylureas. Secondly, the ApoE 
genotype information was lacking; thus, associations pertinent only to patients carrying $\varepsilon 4$ genotype could have been diluted. Lastly, we had no information on the reason for medication dispensation and no access to further blood or CSF biochemistry that would supplement the matching strategy; thus, residual confounding or confounding by indication cannot be ruled out.

The study's main strength comprises the large cohort of patients with diabetes and established dementia, a typically understudied multimorbid population. Moreover, high-quality data were available on medication dispensations allowing multiple exposure models and overall good coverage in the data sources [21, 23, 27-29]. In addition, the breadth of variables allowed extensive PS matching possibilities, decreasing the variability between matched subjects. This was observed even when matching was restricted and the differences in most unmatched variables were balanced as well. Importantly, the inclusion of incident-user and prevalent-user analyses, drug vs drug comparisons, and inverse-probability weighting provided comprehensive information about the use of antidiabetics and their association to a well-established global cognitive screening test-MMSE.

\section{Conclusions}

In conclusion, there are significant differences between the common antidiabetic medications and their association with changes in MMSE scores. Specifically, metformin and the incretin-based medications should be further evaluated for possible cognitive properties.

\begin{abstract}
Abbreviations
AD: Alzheimer's disease; ATC: Anatomical Therapeutic and Chemical; DPP-4i: Dipeptidyl-peptidase-4 inhibitors; GLP-1a: Glucagon-like peptide-1 analogues; GLD: Glucose-lowering drugs; LISA: Longitudinal integrated database for health insurance and labour market studies; MMSE: Mini-Mental State Examination; MixDem: Mixed-pathology dementia; SGLT-2i: Sodium-glucose co-transporter-2 inhibitors; SMDs: Standardized mean differences; SveDem: Swedish Dementia Registry; Patient Register: Swedish National Patient Register; Death Register: Swedish Cause of Death Register; TZD: Thiazolidinediones.
\end{abstract}

\section{Supplementary Information}

The online version contains supplementary material available at https://doi. org/10.1186/s13195-021-00934-0.

Additional file 1: Supplementary Algorithm 1. Diabetes types assessed from the Patient and Drug Register data.

Additional file 2: Supplementary Figure 1. Study sample selection. DPP-4i, dipeptidyl-peptidase-4 inhibitors; IPW, inverse-probability weighting; MMSE, Mini-Mental State Examination; SveDem, Swedish Dementia Registry; TZD, thiazolidinediones.

Additional file 3: Supplementary Table 1. Balance in baseline characteristics among incident users versus non-users of antidiabetic medications propensity-score matched cohorts. ChEl, cholinesterase inhibitors; DPP-4i, dipeptidyl-peptidase-4 inhibitors; MMSE, Mini-Mental State Examination; GLDs, glucose-lowering drugs apart from insulin; SMD, standardized mean differences; Comparisons per baseline incident exposure assignment - exposure assessed at one-year period prior to dementia diagnosis in subjects without history of exposure before the one-year period; p-values refer to the exposure "Yes" vs exposure "No" comparisons; Age is described as mean (SD); Charlson comorbidity index, Diabetes duration and MMSE are described as median (IQR); all other variables are described as $n(\%)$; SMDs were calculated for the matching variables; All cohorts matched using 1:4 ratio; "Total eligible" expresses the number of eligible subjects for propensity-score matching from the original cohort, with \% retained after PS matching.

Additional file 4: Supplementary Table 2. Dropout rate and number of follow-ups in observed and imputed data by users of antidiabetic medications. DPP-4i, dipeptidyl-peptidase-4 inhibitors; TZD, thiazolidinediones; Dropout was counted when the patient had neither died nor did the study end occur in the year following the last observed MMSE; Number of follow-ups are expressed in number of follow-ups in total per drug user, and percentage from baseline number of users; Imputed follow-ups were based on the slope of change between observed MMSE measurements and time from baseline.

Additional file 5: Supplementary Table 3. Baseline differences in the propensity-score matched comparison pairs of the non-metformin antidiabetic drug users. AD, Alzheimer's disease; ChEl, cholinesterase inhibitors; DPP-4i, dipeptidyl-peptidase-4 inhibitors; MixDem, mixed-pathology dementia; MMSE, Mini-Mental State Examination; GLDs, glucose-lowering drugs apart from insulin; SMD, standardized mean differences; SMDs were calculated for the matching variables; Matching was restricted by number of available users; Insulin vs sulfonylurea analysis was matched using 1:1 ratio, otherwise 1:4 ratio was used; "Total eligible" expresses the number of eligible subjects for propensity-score matching from the original cohort, with \% retained after PS matching.

\section{Acknowledgements}

We would like to thank all patients, care providers, and staff for providing the information to the Swedish Dementia Registry.

\section{Authors' contributions}

H.X. participated on data preparation and sensitivity analyses. E.S. co-designed the study. N.H. suggested the statistical methods and contributed to the discussion. D.H. advised on methodology. M.A. and B.W. contributed to the introduction and discussion. M.E. interpreted the data and contributed to the discussion. S.G-P. obtained the data. D.R. supervised and co-designed the study. J.S. designed the study, analyzed the data and wrote the manuscript. All authors have revised and contributed to the manuscript's content and approved of its final version.

\section{Funding}

Open access funding provided by Karolinska Institute. This study has been supported by the Swedish Brain Power, Swedish Research Council (grant numbers 2012-2291, 2016-02317, 2018-02843), Alzheimerfonden, Stockholm County Council (ALF project), the Swedish Associations of Local Authorities and Regions, the Swedish Order of Saint John/Johanniterorden, Swedish Society for Medical Research, FORTE (the Swedish Council for Health, Working Life and Welfare, dnr: 2017-01646), the Swedish Stroke Association, Margaretha af Ugglas foundation, and the Stiftelsen för Gamla Tjänarinnor. The supporting bodies played no role in the design, analysis, or interpretation of the data.

\section{Availability of data and materials}

No data are available. The entities responsible for the original data and the Swedish law do not allow for sharing of the data from the Swedish national or quality-of-care registers.

\section{Declarations}

\section{Ethics approval and consent to participate}

The researchers were provided only with anonymized data linkages, and no link could be made to an individual. Analyses were performed only on a group level. The study complies with the Declaration of Helsinki and was approved by the regional ethical committee in Stockholm, Sweden (number of the 
ethical approval: 2017/501-31). Patients were informed about the registration to SveDem, and they have the option to have their data removed from the register. The Swedish law does not require informed consent for registry-based research; however, the regional ethical committees may require informed consent on patients' behalf for any study —no such request was made for this particular study.

\section{Consent for publication}

Not applicable

\section{Competing interests}

The authors declare that they have no competing interests.

\section{Author details}

'Division of Clinical Geriatrics, Center for Alzheimer Research, Department of Neurobiology, Care Sciences and Society, Karolinska Institutet, Neo, Blickagången 16, 14152 Huddinge, Sweden. ${ }^{2}$ Department of Neurology, Second Faculty of Medicine, Charles University, Motol University Hospital, Prague, Czech Republic. ${ }^{3}$ Department of Medical Epidemiology and Biostatistics, Karolinska Institutet, Stockholm, Sweden. ${ }^{4}$ Institute of Environmental Medicine, Karolinska Institutet, Stockholm, Sweden. ${ }^{5}$ Growth and Metabolism, Department of Molecular Medicine and Surgery, Karolinska Institutet, Stockholm, Sweden. ${ }^{6}$ Division of Neurogeriatrics, Center for Alzheimer Research, Department of Neurobiology, Care Sciences and Society, Karolinska Institutet, Stockholm, Sweden. ${ }^{7}$ Theme Aging, Karolinska University Hospital, Huddinge, Sweden.

\section{Received: 13 September 2021 Accepted: 9 November 2021}

Published online: 02 December 2021

\section{References}

1. Bunn F, Burn AM, Goodman C, Rait G, Norton S, Robinson L, et al. Comorbidity and dementia: a scoping review of the literature. BMC Med. 2014;12:192.

2. Biessels GJ, Despa F. Cognitive decline and dementia in diabetes mellitus: mechanisms and clinical implications. Nat Rev Endocrinol. 2018;14:591-604

3. Campbell JM, Stephenson MD, de Courten B, Chapman I, Bellman SM, Aromataris E. Metformin use associated with reduced risk of dementia in patients with diabetes: a systematic review and meta-analysis. J Alzheimers Dis. 2018;65(4):1225-36.

4. Wu CY, Ouk M, Wong YY, Anita NZ, Edwards JD, Yang P, et al. Relationships between memory decline and the use of metformin or DPP4 inhibitors in people with type 2 diabetes with normal cognition or Alzheimer's disease, and the role APOE carrier status. Alzheimers Dement. 2020;16:1663-73.

5. Imfeld P, Bodmer M, Jick SS, Meier CR. Metformin, other antidiabetic drugs, and risk of Alzheimer's disease: a population-based case-control study. J Am Geriatr Soc. 2012;60(5):916-21.

6. Liu Q, Li S, Quan H, Li J. Vitamin B12 status in metformin treated patients: systematic review. PLoS One. 2014;9(6):e100379.

7. Arnold SE, Arvanitakis Z, Macauley-Rambach SL, Koenig AM, Wang HY, Ahima RS, et al. Brain insulin resistance in type 2 diabetes and Alzheime disease: concepts and conundrums. Nat Rev Neurol. 2018;14(3):168-81.

8. Craft S, Raman R, Chow TW, Rafii MS, Sun CK, Rissman RA, et al. Safety, efficacy, and feasibility of Intranasal Insulin for the treatment of mild cognitive impairment and Alzheimer disease dementia: A Randomized Clinical Trial. JAMA Neurol. 2020;77:1099-109.

9. Cukierman-Yaffe T, Bosch J, Diaz R, Dyal L, Hancu N, Hildebrandt P, et al. Effects of basal insulin glargine and omega-3 fatty acid on cognitive decline and probable cognitive impairment in people with dysglycaemia: a substudy of the ORIGIN trial. Lancet Diabetes Endocrinol. 2014;2(7):562-72.

10. Wium-Andersen IK, Osler M, Jorgensen MB, Rungby J, Wium-Andersen MK. Antidiabetic medication and risk of dementia in patients with type 2 diabetes: a nested case-control study. Eur J Endocrinol. 2019;181(5):499-507.
11. Orkaby AR, Cho K, Cormack J, Gagnon DR, Driver JA. Metformin vs sulfonylurea use and risk of dementia in US veterans aged $>/=65$ years with diabetes. Neurology. 2017;89(18):1877-85.

12. Davies MJ, D'Alessio DA, Fradkin J, Kernan WN, Mathieu C, Mingrone $\mathrm{G}$, et al. Management of hyperglycaemia in type 2 diabetes, 2018. A consensus report by the American Diabetes Association (ADA) and the European Association for the Study of Diabetes (EASD). Diabetologia. 2018;61(12):2461-98.

13. Whitmer RA, Karter AJ, Yaffe K, Quesenberry CP Jr, Selby JV. Hypoglycemic episodes and risk of dementia in older patients with type 2 diabetes mellitus. JAMA. 2009;301(15):1565-72.

14. Punthakee Z, Miller ME, Launer LJ, Williamson JD, Lazar RM, CukiermanYaffee T, et al. Poor cognitive function and risk of severe hypoglycemia in type 2 diabetes: post hoc epidemiologic analysis of the ACCORD trial. Diabetes Care. 2012;35(4):787-93.

15. American Diabetes A 12. Older adults: standards of medical care in diabetes-2020. Diabetes Care. 2020;43(Suppl 1):S152-S62.

16. Gold M, Alderton C, Zvartau-Hind M, Egginton S, Saunders AM, Irizarry $M$, et al. Rosiglitazone monotherapy in mild-to-moderate Alzheimer's disease: results from a randomized, double-blind, placebo-controlled phase III study. Dement Geriatr Cogn Disord. 2010;30(2):131-46.

17. Gejl M, Gjedde A, Egefjord L, Moller A, Hansen SB, Vang K, et al. In Alzheimer's disease, 6-month treatment with GLP-1 analog prevents decline of brain glucose metabolism: randomized, placebo-controlled, doubleblind clinical trial. Front Aging Neurosci. 2016;8:108.

18. Grieco M, Giorgi A, Gentile MC, d'Erme M, Morano S, Maras B, et al. Glucagon-like peptide-1: a focus on neurodegenerative d. Front Neurosci. 2019;13:1112

19. Isik AT, Soysal P, Yay A, Usarel C. The effects of sitagliptin, a DPP-4 inhibitor on cognitive functions in elderly diabetic patients with or without Alzheimer's disease. Diabetes Res Clin Pract. 2017;123:192-8.

20. Biessels GJ, Verhagen C, Janssen J, van den Berg E, Zinman B, Rosenstock J, et al. Effect of linagliptin on cognitive performance in patients with type 2 diabetes and cardiorenal comorbidities: the CARMELINA randomized trial. Diabetes Care. 2019;42(10):1930-8.

21. Religa D, Fereshtehnejad SM, Cermakova P, Edlund AK, Garcia-Ptacek S, Granqvist N, et al. SveDem, the Swedish Dementia Registry - a tool for improving the quality of diagnostics, treatment and care of dementia patients in clinical practice. PLoS One. 2015;10(2):e0116538.

22. Eriksdotter M, Kåwe K, Mattsson UB, Nilsson A, Mayer-Standar S, Nägga K, et al. Svenska Demensregister Årsrapport 2018 Accessible online: https:// www.ucr.uu.se/svedem/om-svedem/arsrapporter/svedem-arsrapport 2018/viewdocument/967. Svenska Demensregistret; 2019.

23. Ludvigsson JF, Andersson E, Ekbom A, Feychting $M$, Kim JL, Reuterwall $C$, et al. External review and validation of the Swedish national inpatient register. BMC Public Health. 2011;11:450.

24. World Health Organization. The International Classification of Diseases 10th revision (ICD-10). 1993.

25. Charlson ME, Pompei P, Ales KL, MacKenzie CR. A new method of classifying prognostic comorbidity in longitudinal studies: development and validation. J Chronic Dis. 1987:40(5):373-83.

26. Quan H, Sundararajan V, Halfon P, Fong A, Burnand B, Luthi JC, et al. Coding algorithms for defining comorbidities in ICD-9-CM and ICD-10 administrative data. Med Care. 2005;43(11):1130-9.

27. Ludvigsson JF, Svedberg P, Olen O, Bruze G, Neovius M. The longitudinal integrated database for health insurance and labour market studies (LISA) and its use in medical research. Eur J Epidemiol. 2019;34(4):423-37.

28. Wettermark B, Hammar N, Fored CM, Leimanis A, Otterblad Olausson P, Bergman U, et al. The new Swedish Prescribed Drug Register--opportunities for pharmacoepidemiological research and experience from the first six months. Pharmacoepidemiol Drug Saf. 2007;16(7):726-35.

29. Brooke HL, Talback M, Hornblad J, Johansson LA, Ludvigsson JF, Druid $\mathrm{H}$, et al. The Swedish cause of death register. Eur J Epidemiol. 2017:32(9):765-73.

30. Handels R, Jonsson L, Garcia-Ptacek S, Eriksdotter M, Wimo A. Controlling for selective dropout in longitudinal dementia data: application to the SveDem registry. Alzheimers Dement. 2020;16(5):789-96.

31. R Core Team. R: A language and environment for statistical computing. $R$ Foundation for Statistical Computing, Vienna, Austria. 2020. URL https:// www.R-project.org/. 
32. Scherrer JF, Salas J, Floyd JS, Farr SA, Morley JE, Dublin S. Metformin and sulfonylurea use and risk of incident dementia. Mayo Clin Proc. 2019;94(8):1444-56.

33. Bohlken J, Jacob L, Kostev K. Association between the use of antihyperglycemic drugs and dementia risk: a case-control study. J Alzheimers Dis. 2018;66(2):725-32.

34. Hettich MM, Matthes F, Ryan DP, Griesche N, Schroder S, Dorn S, et al. The anti-diabetic drug metformin reduces BACE1 protein level by interfering with the MID1 complex. PLoS One. 2014;9(7):e102420.

35. Gupta A, Bisht B, Dey CS. Peripheral insulin-sensitizer drug metformin ameliorates neuronal insulin resistance and Alzheimer's-like changes. Neuropharmacology. 2011;60(6):910-20.

36. Lu XY, Huang $S$, Chen QB, Zhang D, Li W, Ao R, et al. Metformin ameliorates abeta pathology by insulin-degrading enzyme in a transgenic mouse model of Alzheimer's disease. Oxidative Med Cell Longev. 2020;2020:2315106

37. Chen Y, Zhou K, Wang R, Liu Y, Kwak YD, Ma T, et al. Antidiabetic drug metformin (GlucophageR) increases biogenesis of Alzheimer's amyloid peptides via up-regulating BACE1 transcription. Proc Natl Acad Sci U S A. 2009;106(10):3907-12.

38. Son SM, Shin HJ, Byun J, Kook SY, Moon M, Chang YJ, et al. Metformin facilitates amyloid-beta generation by beta- and gamma-secretases via autophagy activation. J Alzheimers Dis. 2016;51(4):1197-208.

39. Secnik J, Xu H, Schwertner E, Hammar N, Alvarsson M, Winblad B, et al Dementia diagnosis is associated with changes in antidiabetic drug prescription: an open-cohort study of approximately 130,000 Swedish subjects over 14 years. J Alzheimers Dis. 2020;76(4):1581-94.

40. Chen KC, Chung CH, Lu CH, Tzeng NS, Lee CH, Su SC, et al. Association between the use of dipeptidyl peptidase 4 inhibitors and the risk of dementia among patients with type 2 diabetes in Taiwan. J Clin Med. 2020:9(3):660

41. Rizzo MR, Barbieri M, Boccardi V, Angellotti E, Marfella R, Paolisso G. Dipeptidyl peptidase-4 inhibitors have protective effect on cognitive impairment in aged diabetic patients with mild cognitive impairment. J Gerontol A Biol Sci Med Sci. 2014;69(9):1122-31.

42. Borzi AM, Condorelli G, Biondi A, Basile F, Vicari ESD, Buscemi C, et al. Effects of vildagliptin, a DPP-4 inhibitor, in elderly diabetic patients with mild cognitive impairment. Arch Gerontol Geriatr. 2019;84:103896.

43. Cukierman-Yaffe T, Bosch J, Jung H, Punthakee Z, Gerstein HC. Hypoglycemia and incident cognitive dysfunction: a post hoc analysis from the ORIGIN trial. Diabetes Care. 2019;42(1):142-7.

44. Morales J. The pharmacologic basis for clinical differences among GLP-1 receptor agonists and DPP-4 inhibitors. Postgrad Med. 2011;123(6):189-201.

45. Kosaraju J, Holsinger RMD, Guo L, Tam KY. Linagliptin, a dipeptidyl peptidase-4 inhibitor, mitigates cognitive deficits and pathology in the 3xTg-AD mouse model of Alzheimer's disease. Mol Neurobiol. 2017;54(8):6074-84.

46. Athauda D, Foltynie T. Insulin resistance and Parkinson's disease: a new target for disease modification? Prog Neurobiol. 2016;145-146:98-120.

47. Andrews JS, Desai U, Kirson NY, Zichlin ML, Ball DE, Matthews BR. Disease severity and minimal clinically important differences in clinical outcome assessments for Alzheimer's disease clinical trials. Alzheimers Dement ( Y). 2019;5:354-63.

48. Weinstein G, Davis-Plourde KL, Conner S, Himali JJ, Beiser AS, Lee A, et al. Association of metformin, sulfonylurea and insulin use with brain structure and function and risk of dementia and Alzheimer's disease: pooled analysis from 5 cohorts. PLoS One. 2019;14(2):e0212293.

49. National Board of Health and Welfare / Socialstyrelsen: National Guidelines for Diabetes Care. Support for governance and management / Nationella Riktlinjer för Diabetesvård. Stöd för Styrning och Ledning Accessible online: https://www.socialstyrelsen.se/globalassets/share point-dokument/artikelkatalog/nationella-riktlinjer/2018-10-25.pdf20 18:[135 p.].

50. Takeda and Zinfandel Pharmaceuticals discontinue TOMMORROW trial following planned futility analysis. [press release]. 2018; Available from: https://www.takeda.com/newsroom/newsreleases/2018/takeda-tommo rrow-trial/.

\section{Publisher's Note}

Springer Nature remains neutral with regard to jurisdictional claims in published maps and institutional affiliations.
Ready to submit your research? Choose BMC and benefit from:

- fast, convenient online submission

- thorough peer review by experienced researchers in your field

- rapid publication on acceptance

- support for research data, including large and complex data types

- gold Open Access which fosters wider collaboration and increased citations

- maximum visibility for your research: over $100 \mathrm{M}$ website views per year

At BMC, research is always in progress.

Learn more biomedcentral.com/submissions 